\title{
NUTRIÇÃO MINERAL DE POPULAÇÕES E HÍBRIDOS DE COQUEIRO (Cocos nucifera -L.) CULTIVADOS EM BEBEDOURO (SP) ${ }^{1}$
}

\author{
LUIZ ANTONIO JUNQUEIRA TEIXEIRA² \& JOSÉ ANTÔNIO ALBERTO DA SILVA ${ }^{3}$
}

RESUMO - Avaliou-se o estado nutricional de sete genótipos de coqueiro (cinco populações: Anão Amarelo de Gramame, Anão Amarelo da Malásia, Anão Vermelho de Gramame, Anão Verde do Jiqui e Gigante Brasileiro da Praia do Forte e dois híbridos: Anão Amarelo de Gramame x Gigante do Oeste Africano e Anão Vermelho de Gramame x Gigante Brasileiro da Praia do Forte), com 2 anos e 4 meses de idade, plantados em Bebedouro (SP). Com o objetivo de monitorar o estado nutricional das plantas e determinar os efeitos do cultivo sobre a fertilidade do solo, analisaram-se tecido vegetal de folhas com idades diferentes (folhas 4 e 9) e solo. Amostras de folha 4, em comparação com as de folha 9, foram mais eficientes para discriminar as populações e híbridos de coqueiro quanto ao seu estado nutricional. As maiores limitações nutricionais dos genótipos em estudo foram as de zinco, potássio, nitrogênio e cobre. Aparentemente, todas as populações e híbridos observados estavam adequadamente nutridos com fósforo, cálcio, boro, ferro e manganês. Como medida mais urgente para a melhoria do estado nutricional das plantas, recomenda-se aumentar a aplicação de Zn. Os principais efeitos do cultivo de coqueiro sobre a fertilidade relacionaram-se com a acidificação do solo na área de aplicação de adubos.

Termos para indexação: nitrogênio, fósforo, potássio, cálcio, magnésio, boro, cobre, ferro, manganês, zinco, análise foliar, coco, análise de solo

\section{MINERAL NUTRITION OF POPULATIONS AND HYBRIDS OF COCONUT (Cocos nucifera L.) GROWN IN BEBEDOURO (SP), BRAZIL}

ABSTRACT - Nutritional status of seven coconut genotypes (Anão Amarelo de Gramame, Anão Amarelo da Malásia, Anão Vermelho de Gramame, Anão Verde do Jiqui, Gigante Brasileiro da Praia do Forte, Anão Amarelo de Gramame x Gigante do Oeste Africano, and Anão Vermelho de Gramame x Gigante Brasileiro da Praia do Forte) were assessed. Different aged leaves (leaf 4 and 9) and soil were sampled with the objective of investigating nutritional status of plants and changes on soil fertility. Samples from leaf 4 were more efficient to distinguish genotypes according to their nutritional conditions than leaf 9 samples. The most serious disorders were zinc, potassium, nitrogen and copper deficiencies. P, Ca, B, Fe and Mn leaf content seemed to be adequate in all genotypes. It was recommended to increase $\mathrm{Zn}$ application rate in order to improve nutritional status. The most outstanding effects of cropping on soil fertility were due to acidification in the fertilizer spreading area.

Index terms: nitrogen, phosphorus, potassium, calcium, magnesium, boron, copper, iron, manganese, zinc, leaf analysis, soil analysis

O cultivo de coco no Brasil apresenta duas realidades bastante distintas. De um lado, têm-se os plantios destinados à produção de coco seco, geralmente com baixa rentabilidade, o que, nos últimos anos, limitou a expansão dessa atividade e, de outro, áreas visando à produção de coco verde, que tiveram um grande incentivo devido ao mercado crescente para água de coco.

O estado nutricional dos coqueiros tem efeito sobre seu desenvolvimento vegetativo, além de determinar, em grande parte, o volume e a qualidade da produção. O acompanhamento do estado nutricional da cultura por meio de análises de tecido foliar foi apontado por Sobral \& Santos (1987) como ferramenta auxiliar para a recomendação de adubação. Segundo Rognon (1984), o conteúdo de nutrientes no tecido foliar de coqueiros pode variar em função do tipo de planta (gigante ou híbridos).

Atualmente, o conhecimento sobre nutrição de coqueiros, bem como adubação recomendada para a cultura, baseia-se na experiência de plantios conduzidos no exterior ou na região Nordeste, já que, em São Paulo, estudos desta natureza estão em fase inicial. Assim, o objetivo deste trabalho foi monitorar o estado nutricional de cinco populações e dois híbridos de coqueiro, assim como os efeitos do cultivo sobre a fertilidade do solo.

$\mathrm{O}$ experimento foi estabelecido na Estação Experimental de Citricultura de Bebedouro (EECB)-SP (lat.: $20^{\circ} 53^{\prime}$ S, long.: $48^{\circ} 28^{\prime} \mathrm{W}$ e altitude: 601 m), em 30/nov/1999, num Latossolo Vermelho Distrófico A moderado textura média, com clima mesotérmico de inverno seco (Cwaclassificação de Köeppen). Alguns atributos químicos do solo obtidos em amostragem prévia ao plantio são apresentados na Tabela 1.

Os genótipos estudados fazem parte de um experimento da Rede Nacional de Avaliação de Cultivares de Coqueiro, realizado em parceria da EECB com a EMBRAPA-CPATC. Os sete tratamentos constituíram-se por cinco populações (Anão Amarelo de Gramame - AAG, Anão Amarelo da Malásia - AAM, Anão Vermelho de Gramame - AVG, Anão Verde do Jiqui - AVJ e Gigante Brasileiro da Praia do Forte GBrPF) e dois híbridos (Anão Amarelo de Gramame x Gigante do Oeste Africano - AAG - - -GOA e Anão Vermelho de Gramame x Gigante Brasileiro da Praia do Forte-AVG -x -GBrPF).

As parcelas experimentais continham seis plantas, com espaçamento de $8 \times 7 \mathrm{~m}$, dispostas em blocos ao acaso, com quatro repetições. As plantas foram irrigadas por microaspersão e adubadas de acordo com cronograma da Tabela 2.

O estado nutricional das plantas está sendo monitorado por análise química de tecido foliar, com amostragens efetuadas duas vezes ao ano (abril - fim da estação das chuvas, e outubro - fim da seca), coletando-se folíolos das folhas 4 e 9 . A amostragem seguiu os procedimentos descritos por Rognon (1984), que recomenda a coleta de folíolos da folha 4 para plantas de genótipos híbridos com menos de 3 anos e da

TABELA 1 - Análise química do solo da área experimental. Valores médios de matéria orgânica, $\mathrm{pH}$ em $\mathrm{CaCl}_{2}, \mathrm{P} \mathrm{K}^{+}, \mathrm{Ca}^{2+}, \mathrm{Mg}^{2+}, \mathrm{H}+\mathrm{Al}$, soma de bases (SB), capacidade de troca de cátions $(\mathrm{T})$ e saturação por bases $(\mathrm{V})$ de amostras coletadas em três profundidades antes da implantação do experimento. Bebedouro (SP), julho/1999.

\begin{tabular}{|c|c|c|c|c|c|c|c|c|c|c|}
\hline Camada & $\mathrm{MO}$ & $\mathrm{pH}^{(1)}$ & $\mathrm{P}^{(2)}$ & $\mathrm{K}^{+}$ & $\mathrm{Ca}^{2+}$ & $\mathrm{Mg}^{2+}$ & $\mathrm{H}+\mathrm{Al}$ & SB & $\mathrm{T}$ & $\mathrm{V}$ \\
\hline ------ cm ------ & $\mathrm{g} \mathrm{dm}^{-3}$ & & $\mathrm{mg} \mathrm{dm} \mathrm{dm}^{-3}$ & ---- & - & $1_{c} \mathrm{dm}^{-3}$ & ------ & ----- & ---- & $---\% \quad--$ \\
\hline $0-20$ & 18 & 4,7 & 6 & 1,3 & 9 & 4 & 40 & 14,3 & 54,3 & 26 \\
\hline $20-40$ & 16 & 4,4 & 3 & 0,6 & 6 & 3 & 42 & 9,6 & 51,6 & 19 \\
\hline $40-60$ & 16 & 4,4 & 4 & 0,6 & 7 & 4 & 45 & 11,6 & 56,6 & 20 \\
\hline
\end{tabular}

${ }^{(1)} \mathrm{pH}$ em $\mathrm{CaCl}_{2}(0,01 \mathrm{M}) .{ }^{(2)}$ Extração com resina trocadora de íons.

\footnotetext{
${ }_{1}^{1}$ (Trabalho 100/2002). Recebido: 15/07/2002. Aceito para publicação: 11/07/2003.

${ }^{2}$ Pesquisador Científico do CAPTA-Frutas/Instituto Agronômico. Cx. Postal 28. 13001-970, Campinas-SP. teixeira@iac.sp.gov.br.

${ }^{3}$ Pesquisador Científico da Estação Experimental de Citricultura de Bebedouro-SP.
} 
TABELA 2 - Cronograma de aplicação de corretivos e adubos no experimento. Bebedouro (SP), julho de 2002.

\begin{tabular}{|c|c|c|}
\hline Data & Corretivo/adubo & Dose \\
\hline ago/99 & Calcário dolomítico & Correção para $V=70 \%$ \\
\hline nov/99 & Superfosfato simples & $500 \mathrm{~g} / \mathrm{cova}$ \\
\hline$"$ & Calcário dolomítico & $500 \mathrm{~g} /$ cova \\
\hline " & Esterco de curral (curtido) & $20 \mathrm{~L} /$ cova \\
\hline $\mathrm{fev} / 00$ & Nitrocálcio & $150 \mathrm{~g} /$ planta \\
\hline $\mathrm{abr} / 00$ & Nitrocálcio & $150 \mathrm{~g} /$ planta \\
\hline jun/00 & $12-6-12$ & $300 \mathrm{~g} /$ planta \\
\hline$"$ & Ácido bórico & $20 \mathrm{~g} /$ planta \\
\hline ago/00 & $12-6-12$ & $300 \mathrm{~g} /$ planta \\
\hline$"$ & Superfosfato simples & $150 \mathrm{~g} / \mathrm{planta}$ \\
\hline$"$ & Nitrocálcio & $100 \mathrm{~g} /$ planta \\
\hline ” & Foliar (Zn, B e Mn) & (1) \\
\hline out $/ 00$ & $12-6-12$ & $300 \mathrm{~g} /$ planta \\
\hline$"$ & Superfosfato simples & $150 \mathrm{~g} /$ planta \\
\hline$"$ & Nitrocálcio & $100 \mathrm{~g} /$ planta \\
\hline " & Foliar (Zn, B e Mn) & (1) \\
\hline $\operatorname{dez} / 00$ & $12-6-12$ & $300 \mathrm{~g} /$ planta \\
\hline " & Superfosfato simples & $150 \mathrm{~g} /$ planta \\
\hline ” & Nitrocálcio & $100 \mathrm{~g} /$ planta \\
\hline$"$ & Foliar (Zn, B e Mn) & (1) \\
\hline $\mathrm{fev} / 01$ & $12-6-12$ & $500 \mathrm{~g} /$ planta \\
\hline & Ácido bórico & $20 \mathrm{~g} / \mathrm{planta}$ \\
\hline $\mathrm{abr} / 01$ & $12-6-12$ & $500 \mathrm{~g} /$ planta \\
\hline$"$ & Ácido bórico & $20 \mathrm{~g} / \mathrm{planta}$ \\
\hline jun/01 & $12-6-12$ & $500 \mathrm{~g} /$ planta \\
\hline$"$ & Ácido bórico & $20 \mathrm{~g} / \mathrm{planta}$ \\
\hline ago/01 & $12-6-12$ & $500 \mathrm{~g} /$ planta \\
\hline . & Ácido bórico & $20 \mathrm{~g} /$ planta \\
\hline out/01 & $12-6-12$ & $500 \mathrm{~g} / \mathrm{planta}$ \\
\hline ” & Ácido bórico & $20 \mathrm{~g} /$ planta \\
\hline " & Foliar (Zn, B e Mn) & (1) \\
\hline $\mathrm{dez} / 01$ & $12-6-12$ & $500 \mathrm{~g} /$ planta \\
\hline$"$ & Ácido bórico & $20 \mathrm{~g} /$ planta \\
\hline $\mathrm{fev} / 02$ & $12-6-12$ & $500 \mathrm{~g} /$ planta \\
\hline$"$ & Ácido bórico & $20 \mathrm{~g} /$ planta \\
\hline $\mathrm{abr} / 02$ & $12-6-12$ & $500 \mathrm{~g} / \mathrm{plan}$ ta \\
\hline$"$ & Ácido bórico & $20 \mathrm{~g} /$ planta \\
\hline jun/02 & $12-6-12$ & $500 \mathrm{~g} /$ planta \\
\hline & Ácido bórico & $20 \mathrm{~g} /$ planta \\
\hline
\end{tabular}

(1) $8 \mathrm{~L} /$ planta de uma solução com $0,014 \%$ de $\mathrm{Zn}, 0,015 \%$ de B e $0,025 \%$ de Mn.

folha 9, para plantas com idade entre 3 e 4 anos. O tecido foliar foi analisado quanto aos teores de macro e micronutrientes de interesse agrícola, de acordo com Bataglia et al. (1983). Neste trabalho, serão apresentados os resultados referentes à primeira amostragem realizada em 25/abr/2002, dois anos e quatro meses após o plantio. Nesta data, foram coletadas amostras de solo na projeção da copa (área de aplicação de fertilizantes) e entre as linhas de plantio, das camadas de 0 a $20 \mathrm{~cm}$ e de 20 a $40 \mathrm{~cm}$, analisadas segundo Raij \& Quaggio (1983).

A distribuição dos nutrientes no dossel das plantas foi estimada por meio da comparação dos teores foliares obtidos em amostragens de folhas com idades diferentes; folha 4, emitida mais recentemente, e folha 9 , mais antiga. $\mathrm{O}$ estado nutricional das plantas foi avaliado comparando-se os teores foliares das amostras com os níveis críticos apresentados por Rognon (1984) e Sobral (1998). O efeito do cultivo sobre o solo foi estimado comparando-se amostras coletadas sob a projeção da copa com as das entrelinhas. Os resultados foram analisados empregando-se módulo GLM (General linear models) do SAS, segundo Freund \& Littell (1981).

Apesar de as plantas estarem com pouco mais de dois anos, algumas consequiências do cultivo de coqueiros sobre atributos químicos do solo podem ser identificadas nas Tabelas 3 e 4. Comparando-se as amostras retiradas na projeção da copa (área de aplicação dos adubos) com as obtidas nas entre=linhas, observam-se valores de $\mathrm{pH}$, saturação por bases, $\mathrm{Ca}$ e $\mathrm{Mg}$ menores para as primeiras. Os principais efei- tos estão relacionados com a acidificação causada, especialmente, pela aplicação de fertilizantes nitrogenados. Estes resultados são compatíveis com os descritos para outros cultivos como bananeira (Saes, 1995; Teixeira et al., 2001), seringueira (Bataglia \& Santos, 1999) e citros (Sanches et al., 1999).

As estimativas dos teores foliares dos nutrientes foram mais precisas para as amostras de folha 4 (menores erros padrões das médias e coeficientes de variação) do que para as de folha 9 (Tabela 5). A maior precisão, somada à amplitude maior de variação dos teores na folha 4 , possibilitou discriminar melhor os sete genótipos quanto ao seu estado nutricional, especialmente para $\mathrm{P}, \mathrm{Ca}, \mathrm{B}$ e Zn.

Nitrogênio. Em média, os teores de $\mathrm{N}$ na folha 4 foram inferiores ao nível crítico (22 $\left.\mathrm{g} \mathrm{kg}^{-1}\right)$ sugerido por Rognon (1984), não sendo observadas diferenças significativas entre os genótipos (Tabela 5). A dose de $360 \mathrm{~g} /$ planta/ano de N (Tabela 2) fracionada em seis aplicações durante $\mathrm{o}$ ano que antecedeu a amostragem foliar não foi suficiente para atender às necessidades dos diversos genótipos. Para quase todos materiais, o teor de $\mathrm{N}$ foi maior na folha 4, indicando que houve mobilização de $\mathrm{N}$ das folhas mais velhas (Tabela 6). Broschat (1997) observou que, a partir da quarta folha de coqueiro, a concentração de $\mathrm{N}$ diminuiu proporcionalmente à idade das folhas, atribuindo esta variação à mobilidade do elemento na planta.

Potássio. Comparando-se os teores de $\mathrm{K}$ foliar dos sete genótipos com os níveis críticos (NC) apresentados por Rognon (1984), observa-se que, com exceção do GBrPF, para o qual o NC de Ké $17,5 \mathrm{~g} \mathrm{~kg}^{-}$ ${ }^{1}$, os demais apresentaram concentrações de $\mathrm{K}$ foliar inferiores ao NC definido para híbridos (20 $\left.\mathrm{g} \mathrm{kg}^{-1}\right)$ (Tabela 5). A intensa mobilização de potássio das folhas velhas para as novas, em todos os materiais estudados (Tabela 6), e os teores médios detectados na quarta folha são indícios de deficiência nutricional; entretanto, as plantas não apresentaram sintomas de deficiência de K. No ano que antecedeu a amostragem foliar, foram aplicadas $360 \mathrm{~g} /$ planta de $\mathrm{K}_{2} \mathrm{O}$ (Tabela 2) o que, aparentemente, não foi suficiente. Com a emissão dos cachos, caso não seja aumentada a adubação com $\mathrm{K}$, é possível que as folhas mais velhas de certos genótipos manifestem sua falta. A movimentação de $\mathrm{K}$ das folhas velhas para as mais novas é comum em várias espécies vegetais, sendo, geralmente, segundo Broschat (1997), da mesma ordem de grandeza da mobilização de N e menor do que a de P. Ollivier (1993) observou que os sintomas visuais de falta de $\mathrm{K}$ manifestam-se com teores foliares abaixo de $5 \mathrm{~g} \mathrm{~kg}^{-1}$, quando as plantas já estão em processo severo de deficiência nutricional. As amostras de solo coletadas na área mais próxima das plantas apresentaram teores de $\mathrm{K}$ trocável entre baixo e médio (Tabela 3), segundo os limites de interpretação sugeridos por Raij et al. (1996). Mesmo com a aplicação de fertilizantes potássicos, o teor de K trocável na área de projeção da copa não diferiu daquele de amostras das entre= linhas, sugerindo que a extração deste nutriente pelos coqueiros é elevada. Outras culturas exigentes em K, como bananeira, causam significativa redução no teor de $\mathrm{K}$ trocável do solo, como descrito por Teixeira et al. (2001).

Fósforo. Observa-se na Tabela 5 que os sete genótipos apresentaram teores foliares de $\mathrm{P}$ adequados $\left(>1,4 \mathrm{~g} \mathrm{~kg}^{-1}\right)$, com pequenas variações entre eles. A aplicação de $180 \mathrm{~g} /$ planta de $\mathrm{P}_{2} \mathrm{O}_{5}$ durante o ano anterior à amostragem (Tabela 2) foi suficiente para atender às necessidades dos coqueiros. As diferenças entre as amostras das folhas 4 e 9 (Tabela 6) estão de acordo com as observações de Broschat (1997), revelando possível mobilização deste nutriente das folhas velhas para novas. A concentração de $\mathrm{P}$ disponível no solo, na projeção da copa (Tabela 3), classifica-se como média para a camada de 0 a $20 \mathrm{~cm}$, e baixa, para 20 a $40 \mathrm{~cm}$ (Raij et al.,- 1996). Estes valores parecem ser suficientes para suprir as necessidades da cultura, considerada por Khan (1985) como pouco exigente em fósforo.

Cálcio. Os teores de Ca na folha 4 (Tabela 5) indicaram que todos os genótipos estavam suficientemente nutridos com este elemento, pois foram superiores ao nível crítico de $3,4 \mathrm{~g} \mathrm{~kg}^{-1}$, apresentado por Sobral (1998). A maior concentração de Ca nas folhas mais velhas (Tabela 6) foi decorrente de sua baixa mobilidade na planta, o que concorda 
TABELA 3 - Análise química do solo da área experimental. Valores médios de matéria orgânica, pH em $\mathrm{CaCl}_{2}, \mathrm{P}, \mathrm{K}^{+}, \mathrm{H}+\mathrm{Al}$, soma de bases ( $\mathrm{SB}$ ), capacidade de troca de cátions $(\mathrm{T})$ e saturação por bases $(\mathrm{V})$ de amostras coletadas em duas posições e duas profundidades. Bebedouro (SP), maio/2002.

\begin{tabular}{|c|c|c|c|c|c|c|c|c|c|}
\hline Camada & Posição & MO & $\mathrm{pH}^{(3)}$ & $\mathrm{P}^{(4)}$ & $\mathrm{K}^{+}$ & $\mathrm{H}+\mathrm{Al}$ & SB & $\mathrm{T}$ & V \\
\hline ------- cm ------- & & $\mathrm{g} \mathrm{dm}^{-3}$ & & $\mathrm{mg} \mathrm{dm}^{-3}$ & ------- & $---\mathrm{mmc}$ & $-3-$ & ------- & --- \% --- \\
\hline $0-20$ & $\mathrm{PC}^{(1)}$ & $17 \mathrm{a}^{(2)}$ & $5,0 \mathrm{a}$ & $19 \mathrm{a}$ & $1,7 \mathrm{a}$ & $30 \mathrm{a}$ & $29,5 \mathrm{a}$ & $60,0 \mathrm{a}$ & $49 \mathrm{a}$ \\
\hline $0-20$ & EL & $16 \mathrm{~b}$ & $5,5 \mathrm{~b}$ & $11 \mathrm{~b}$ & $1,9 \mathrm{a}$ & $23 \mathrm{~b}$ & $52,5 \mathrm{~b}$ & $75,4 \mathrm{~b}$ & $69 \mathrm{~b}$ \\
\hline $20-40$ & PC & $12 \mathrm{~A}$ & $4,4 \mathrm{~A}$ & $8 \mathrm{~A}$ & $0,8 \mathrm{~A}$ & $42 \mathrm{~A}$ & $10,7 \mathrm{~A}$ & $52,4 \mathrm{~A}$ & $20 \mathrm{~A}$ \\
\hline $20-40$ & EL & $13 \mathrm{~A}$ & $4,9 \mathrm{~B}$ & $6 \mathrm{~A}$ & $1,2 \mathrm{~B}$ & $32 \mathrm{~B}$ & $24,9 \mathrm{~B}$ & $57,2 \mathrm{~A}$ & $43 \mathrm{~B}$ \\
\hline
\end{tabular}

(1) $\mathrm{PC}=$ projeção da copa (área de aplicação de fertilizantes); EL=entrelinha. ${ }^{(2)}$ Médias de uma mesma profundidade de amostragem seguidas por letras iguais não diferem entre si (teste $\mathrm{F}, \mathrm{p}>0,05) .{ }^{(3)} \mathrm{pH}$ em $\mathrm{CaCl}_{2}(0,01 \mathrm{M})$. ${ }^{(4)}$ Extração com resina trocadora de íons.

TABELA 4 - Análise química do solo da área experimental. Valores médios de $\mathrm{Ca}^{2+}, \mathrm{Mg}^{2+}, \mathrm{B}, \mathrm{Cu}, \mathrm{Fe}, \mathrm{Mn}$ e $\mathrm{Zn}$ de amostras coletadas em duas posições e duas profundidades. Bebedouro (SP), maio/2002.

\begin{tabular}{|c|c|c|c|c|c|c|c|c|}
\hline Camada & Posição & $\mathrm{Ca}^{2+}$ & $\mathrm{Mg}^{2+}$ & B & $\mathrm{Cu}$ & $\mathrm{Fe}$ & $\mathrm{Mn}$ & $\mathrm{Zn}$ \\
\hline $\mathrm{cm}$ & & $---m n$ & 3 ---- & ------ & - 1 & . & 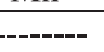 & \\
\hline $0-20$ & $\mathrm{PC}^{(1)}$ & $22 \mathrm{a}^{(2)}$ & $6 \mathrm{a}$ & $0,38 \mathrm{a}$ & $0,8 \mathrm{a}$ & $45 \mathrm{a}$ & $4,0 \mathrm{a}$ & $0,7 \mathrm{a}$ \\
\hline $0-20$ & EL & $31 \mathrm{~b}$ & $19 \mathrm{~b}$ & $0,15 \mathrm{~b}$ & $0,7 \mathrm{a}$ & $33 \mathrm{~b}$ & $2,9 \mathrm{~b}$ & $0,7 \mathrm{a}$ \\
\hline $20-40$ & PC & $8 \mathrm{~A}$ & $2 \mathrm{~A}$ & $0,39 \mathrm{~A}$ & $0,7 \mathrm{~A}$ & $40 \mathrm{~A}$ & $1,7 \mathrm{~A}$ & $0,5 \mathrm{~A}$ \\
\hline $20-40$ & EL & $14 \mathrm{~B}$ & $9 \mathrm{~B}$ & $0,16 \mathrm{~B}$ & $0,6 \mathrm{~A}$ & $32 \mathrm{~B}$ & $1,9 \mathrm{~A}$ & $0,4 \mathrm{~A}$ \\
\hline
\end{tabular}

(1) $\mathrm{PC}=$ projeção da copa (área de aplicação de fertilizantes); EL=entrelinha. ${ }^{(2)}$ Médias de uma mesma profundidade de amostragem seguidas por letras iguais não diferem entre si (teste $F, p>0,05$ ).

TABELA 5 - Teores de nutrientes nas folhas quatro e nove amostradas em sete genótipos de coqueiro aos 28 meses após plantio. Bebedouro (SP), abril/2002.

\begin{tabular}{|c|c|c|c|c|c|c|c|c|c|c|}
\hline Genótipo & $\mathbf{N}$ & $\mathbf{K}$ & $\mathbf{P}$ & $\mathrm{Ca}$ & Mg & B & $\mathrm{Cu}$ & $\mathbf{F e}$ & Mn & $\mathbf{Z n}$ \\
\hline \multirow{2}{*}{\multicolumn{11}{|c|}{$\begin{array}{c}\text {----------------------------- g/kg -------------------- } \\
\end{array}$}} \\
\hline \multicolumn{6}{|c|}{ Folha 4} & & & & & \\
\hline AAG & $20,2 a^{(1)}$ & $17,3 \mathrm{ab}$ & $1,7 \mathrm{a}$ & $3,9 \mathrm{ab}$ & $2,4 a$ & $38,9 \mathrm{ab}$ & $4,1 \mathrm{a}$ & $134 \mathrm{a}$ & $115 \mathrm{a}$ & $11,9 \mathrm{a}$ \\
\hline $\mathbf{A A M}$ & $19,6 \mathrm{a}$ & $15,4 b c$ & $1,6 a b c$ & $3,6 a b$ & $1,9 \mathrm{a}$ & $40,1 \mathrm{ab}$ & $4,1 \mathrm{a}$ & $117 \mathrm{a}$ & $142 \mathrm{a}$ & $10,5 \mathrm{ab}$ \\
\hline AVG & $20,0 \mathrm{a}$ & $13,0 \mathrm{c}$ & $1,5 \mathrm{abc}$ & $3,7 \mathrm{ab}$ & $2,3 \mathrm{a}$ & $46,1 \mathrm{a}$ & $3,2 \mathrm{a}$ & $135 \mathrm{a}$ & $123 a$ & $9,1 \mathrm{ab}$ \\
\hline AVJ & $18,4 \mathrm{a}$ & $12,6 \mathrm{c}$ & $1,5 \mathrm{bc}$ & $3,5 b$ & $2,1 \mathrm{a}$ & $39,5 \mathrm{ab}$ & $2,8 \mathrm{a}$ & $157 \mathrm{a}$ & $129 a$ & $7,9 b$ \\
\hline AAGxGOA & $18,7 \mathrm{a}$ & $14,6 b c$ & $1,5 \mathrm{abc}$ & $3,9 \mathrm{ab}$ & $2,3 \mathrm{a}$ & $38,0 \mathrm{ab}$ & $4,1 \mathrm{a}$ & $131 \mathrm{a}$ & $130 \mathrm{a}$ & $11,1 \mathrm{ab}$ \\
\hline AVGxGBrPF & $19,6 a$ & $14,5 b c$ & $1,4 \mathrm{c}$ & $4,1 \mathrm{ab}$ & $2,1 \mathrm{a}$ & $35,1 \mathrm{ab}$ & $3,7 \mathrm{a}$ & $165 \mathrm{a}$ & $148 \mathrm{a}$ & $10,4 \mathrm{ab}$ \\
\hline GBrPF & $18,8 \mathrm{a}$ & $19,2 \mathrm{a}$ & $1,6 \mathrm{ab}$ & $4,8 \mathrm{a}$ & $2,1 \mathrm{a}$ & $32,2 b$ & $3,8 \mathrm{a}$ & $154 \mathrm{a}$ & $104 a$ & $11,9 \mathrm{a}$ \\
\hline Média & 19,3 & 15,2 & 1,5 & 3,9 & 2,2 & 38,6 & 3,7 & 142 & 127 & 10,4 \\
\hline EPMédia $^{(2)}$ & 0,64 & 0,76 & 0,05 & 0,25 & 0,13 & 2,91 & 0,33 & 13,5 & 17,4 & 0,78 \\
\hline $\mathrm{DMS}^{(3)}$ & 2,99 & 3,54 & 0,22 & 1,18 & 4,67 & 13,61 & 1,56 & 63,1 & 81,4 & 3,65 \\
\hline $\mathrm{CV}(\%)^{(4)}$ & 6,6 & 10,0 & 6,0 & 12,9 & 12,5 & 15,1 & 18,0 & 19,0 & 27,4 & 15,0 \\
\hline \multicolumn{11}{|c|}{ Folha 9} \\
\hline AAG & $17,5 \mathrm{ab}$ & $9,4 \mathrm{ab}$ & $1,4 \mathrm{a}$ & $5,7 \mathrm{a}$ & $1,9 \mathrm{a}$ & $45,9 \mathrm{a}$ & $1,9 a$ & $148 \mathrm{a}$ & $125 \mathrm{a}$ & $6,6 a$ \\
\hline $\mathbf{A A M}$ & $17,5 \mathrm{ab}$ & $12,3 \mathrm{a}$ & $1,4 \mathrm{a}$ & $5,4 a$ & $1,6 \mathrm{a}$ & $45,5 \mathrm{a}$ & $2,0 \mathrm{a}$ & $175 a$ & $184 \mathrm{a}$ & $6,9 a$ \\
\hline AVG & $19,5 \mathrm{a}$ & $7,2 b$ & $1,4 \mathrm{a}$ & $4,6 a$ & $1,7 \mathrm{a}$ & $43,9 \mathrm{a}$ & $2,3 a$ & $313 a$ & $144 a$ & $7,0 \mathrm{a}$ \\
\hline AVJ & $15,8 b$ & $8,8 \mathrm{ab}$ & $1,3 a$ & $4,3 a$ & $1,7 \mathrm{a}$ & $47,9 \mathrm{a}$ & $1,5 \mathrm{a}$ & $209 a$ & $146 a$ & $5,6 a$ \\
\hline AAGxGOA & $17,3 \mathrm{ab}$ & $7,2 b$ & $1,3 \mathrm{a}$ & $5,5 \mathrm{a}$ & $2,0 \mathrm{a}$ & $45,0 \mathrm{a}$ & $2,2 \mathrm{a}$ & $213 a$ & $190 \mathrm{a}$ & $8,1 \mathrm{a}$ \\
\hline AVGxGBrPF & $18,0 \mathrm{ab}$ & $6,9 b$ & $1,3 a$ & $5,3 a$ & $1,7 \mathrm{a}$ & $44,6 \mathrm{a}$ & $1,8 \mathrm{a}$ & $241 \mathrm{a}$ & $211 \mathrm{a}$ & $6,5 \mathrm{a}$ \\
\hline GBrPF & $16,3 a b$ & $7,8 b$ & $1,4 \mathrm{a}$ & $8,3 b$ & $1,8 \mathrm{a}$ & $47,2 \mathrm{a}$ & $1,9 \mathrm{a}$ & $231 \mathrm{a}$ & $183 a$ & $7,6 \mathrm{a}$ \\
\hline Média & 16,3 & 7,8 & 1,4 & 8,3 & 1,8 & 47,2 & 1,9 & 231 & 183 & 7,6 \\
\hline EPMédia & 0,77 & 0,86 & 0,05 & 0,37 & 0,11 & 4,11 & 0,37 & 66,6 & 26,0 & 0,75 \\
\hline DMS & 3,62 & 3,84 & 0,23 & 1,71 & 0,53 & 19,19 & 1,52 & 311,4 & 121,5 & 3,50 \\
\hline $\mathrm{CV}(\%)$ & 8,9 & 17,2 & 7,5 & 13,1 & 12,7 & 18,0 & 32,3 & 61,0 & 30,8 & 21,7 \\
\hline
\end{tabular}

(1) Valores seguidos por letras iguais, de uma mesma folha e coluna, não diferem entre si, pelo teste de Tukey (p>0,05). ${ }^{(2)}$ EPMédia $=$ erro padrão da média. ${ }^{(3)}$ Diferença mínima significativa estimada pelo teste de Tukey $(\propto=0,05) \cdot{ }^{(4)}$ Coeficiente de variação.

com as observações de Sobral (1998). Segundo Broschat (1997), tanto em coqueiros como em tamareiras, a concentração de Ca foliar decresce com a idade das folhas. A situação nutricional das plantas foi coerente com a disponibilidade de Ca no solo sob a projeção da copa (Tabela 4). Os teores de Ca trocável no solo classificam-se como altos (Raij et al., 1996).

Magnésio. Os teores foliares de $\mathrm{Mg}$ (Tabela 4) variaram em torno dos níveis críticos descritos por Sobral (1998), que são de 2,2 e 2,4 $\mathrm{g} \mathrm{kg}^{-1}$ para gigantes e híbridos, respectivamente. Broschat (1997) afirmou que a relação entre concentração foliar de $\mathrm{Mg}$ e idade da folha é variável entre espécies de palmeiras e entre variedades de coqueiro. De acordo com a Tabela 6, apenas o 'GBrPF' não apresentou movimentação significativa de $\mathrm{Mg}$ para as folhas mais novas, indicando que, provavelmente, ele estava suficientemente nutrido com $\mathrm{Mg}$. No solo, sob a projeção da copa, os teores $\mathrm{Mg}$ (Tabela 4) foram classificados, segundo Raij et al. (1996), como médios. A acidificação decorrente da adubação e a perda de cátions, reveladas pelas diferenças entre amostras coletadas na projeção da copa e nas entrelinhas, exigem monitoração periódica da fertilidade do solo e estado nutricional da cultura.

Micronutrientes. As classes de interpretação de teores de 
micronutrientes no solo podem ser aprimoradas quando se conhecem as exigências próprias de cada grupo ou espécie vegetal (Raij et al., 1996). A interpretação dos resultados de análise foliar, com base em níveis críticos, também requer alguns cuidados, pois estes variam em função dos genótipos e condições ambientais, entre outros fatores (Rognon, 1984). Com culturas pouco estudadas, como é o caso do coqueiro, a situação torna-se mais complexa.

Boro. Os teores foliares de B (Tabela 5) foram superiores $10 \mathrm{mg} \mathrm{kg}^{-1}$, que é o nível crítico apresentado por Rognon (1984) e Sobra (1998). Segundo este último autor, o boro é relativamente imóvel, o que se confirma pelos resultados da Tabela 6, pois, em todos os genótipos, não se detectou mobilização deste nutriente para as folhas mais novas A disponibilidade de B no solo (Tabela 4) classifica-se, segundo Raij et al. (1996), como média. No momento, as plantas estão bem nutridas em boro; entretanto, no ano anterior, apresentaram sintomas de deficiência, o que exigiu a aplicação deste nutriente. A última aplicação de boro via foliar foi há cerca de seis meses antes da amostragem de folhas, quando a folha 4 ainda não havia sido emitida, o que também explica os teores mais elevados deste nutriente na folha 9.

TABELA 6 - Estimativa de redistribuição de nutrientes entre folhas de idades diferentes (folhas 4 e 9) amostradas em sete genótipos de coqueiro aos 28 meses após plantio. Bebedouro (SP), abril/2002.

\begin{tabular}{lccccccccccc}
\hline Genótipo & $\mathrm{N}$ & $\mathrm{K}$ & $\mathrm{P}$ & $\mathrm{Ca}$ & $\mathrm{Mg}$ & $\mathrm{B}$ & $\mathrm{Cu}$ & $\mathrm{Fe}$ & $\mathrm{Mn}$ & $\mathrm{Zn}$ \\
\hline & & \multicolumn{1}{c}{ Redistribuição $^{(\mathbf{1})}$} & & & \\
$\mathrm{AAG}$ & $\uparrow$ & $\uparrow$ & $\uparrow$ & $\downarrow$ & $\uparrow$ & $=$ & $\uparrow$ & $=$ & $=$ & $\uparrow$ \\
$\mathrm{AAM}$ & $\uparrow$ & $\uparrow$ & $\uparrow$ & $\downarrow$ & $\uparrow$ & $=$ & $\uparrow$ & $=$ & $=$ & $\uparrow$ \\
$\mathrm{AVG}$ & $=$ & $\uparrow$ & $\uparrow$ & $\downarrow$ & $\uparrow$ & $=$ & $=$ & $\downarrow$ & $\downarrow$ & $\uparrow$ \\
$\mathrm{AVJ}$ & $\uparrow$ & $\uparrow$ & $\uparrow$ & $\downarrow$ & $\uparrow$ & $\downarrow$ & $\uparrow$ & $=$ & $=$ & $\uparrow$ \\
AAGxGOA & $=$ & $\uparrow$ & $\uparrow$ & $\downarrow$ & $\uparrow$ & $=$ & $\uparrow$ & $=$ & $=$ & $\uparrow$ \\
AVGxGBrPF & $\uparrow$ & $\uparrow$ & $\uparrow$ & $\downarrow$ & $\uparrow$ & $\downarrow$ & $\uparrow$ & $=$ & $=$ & $\uparrow$ \\
GBrPF & $\uparrow$ & $\uparrow$ & $\uparrow$ & $\downarrow$ & $=$ & $\downarrow$ & $\uparrow$ & $=$ & $=$ & $\uparrow$
\end{tabular}

(1) $\uparrow$ : Teor do nutriente na folha quatro é maior $(\mathrm{p}<0,05)$ do que na folha nove; $\downarrow$ : teor do nutriente na folha quatro é menor $(\mathrm{p}<0,05)$ do que na folha nove $=$ : variação dos teores entre folha quatro e nove não difere de zero $(p>0,05)$.

Cobre. Para este nutriente, o estado nutricional dos diversos genótipos pode ser um pouco pior do que em relação ao B. Os teores médios de $\mathrm{Cu}$ (Tabela 5) são menores do que o nível crítico (4 a 5 mg kg', segundo Rognon, 1984) e detectou-se significativa mobilização deste nutriente para as folhas mais novas em quase todos os genótipos (Tabela 6). Entretanto, no solo (Tabela 4), os teores de $\mathrm{Cu}$ variaram de médio a altos (Raij et al., 1996).

Ferro. Os teores de Fe (Tabela 5) indicaram que as plantas estão suficientemente nutridas deste elemento, pois são superiores ao nível crítico (40 $\left.\mathrm{mg} \mathrm{kg}^{-1}\right)$ apresentado por Sobral (1998). Sua baixa mobilidade, aliada à condição de suficiência, determinou que não houvesse mobilização de $\mathrm{Fe}$ das folhas velhas para as novas (Tabela 6). O estado nutricional das plantas em relação ao Fe também refletiu sua disponibilidade no solo, onde os teores (Tabela 4) foram superiores a $12 \mathrm{mg} \mathrm{kg}^{-1}$, nível que Raij et al. (1996) consideram como suficiente.

Manganês. O estado nutricional dos sete genótipos em relação a este nutriente foi semelhante ao descrito para o ferro, com as plantas apresentando teores superiores ao nível crítico $\left(100 \mathrm{mg} \mathrm{kg}^{-1}\right)$ e sem aparente redistribuição (Tabelas 5 e 6). No solo (Tabela 4), os teores de Mn enquadram-se na classe de interpretação média (Raij et al., 1996).

Zinco. A concentração de $\mathrm{Zn}$ nas folhas de todos os genótipos (Tabela 5) ficou abaixo do nível crítico de $15 \mathrm{mg} \mathrm{kg}^{-1}$, apresentado por Sobral (1998). Ainda que o Zn seja considerado um nutriente de baixa mobilidade nas plantas, sua concentração na folha 4 foi superior à das folhas mais velhas em todos os genótipos (Tabela 6), indicando possí- vel deficiência nutricional. A movimentação de Zn fica mais significativa, ainda, ao constatar-se que a última aplicação foliar de $\mathrm{Zn}$ se deu antes da emissão da folha 4 (Tabela 2). Os teores de Zn no solo (Tabela 4) estão entre médios e baixos, segundo Raij et al. (1996). A baixa disponibilidade no solo e o estado nutricional das plantas implicam que seja revisto o manejo deste nutriente no experimento.

Em síntese, com estas observações, demonstrou-se que amostras de folha 4, em comparação com as de folha 9, foram mais eficientes para discriminar as populações e híbridos de coqueiro quanto aos seu estado nutricional. Observou-se que as maiores limitações nutricionais dos genótipo em estudo são as de zinco, potássio, nitrogênio e cobre. Aparentemente, todas as populações e híbridos observados estão adequadamente nutridos com fósforo, cálcio, boro, ferro e manganês. Recomenda-se, como medida de correção mais urgente, aumentar o fornecimento de zinco às plantas. Por fim, constatou-se que os principais efeitos do cultivo de coqueiro sobre a fertilidade foram relacionados com a acidificação do solo na área de aplicação de adubos.

\section{REFERÊNCIAS BIBLIOGRÁFICAS}

BATAGLIA, O.C.; FURLANI, A.M.C.; TEIXEIRA, J.P.F.; FURLANI, P.R.; GALLO, J.R. Métodos de análise química de plantas. Campinas: IAC, 1983. 48p. (Boletim Técnico, 78).

BATAGLIA, O.C.; SANTOS, W.R. Efeitos da adubação NPK na fertilidade do solo, nutrição e crescimento da seringueira. Revista Brasileira de Ciência do Solo, Viçosa, v.23, p.881-890, 1999.

BROSCHAT, K. T. Nutrient ditribution, dynamics, and sampling in coconut and Canary date palms. Journal of the American Society of Horticultural Science, New York, v.122, n.6, p.884-890, 1997.

FREUND, R.J.; LITTELL, R.C. SAS for linear models: a guide to the ANOVA and GLM procedures. Cary: SAS Institute, 1981.231p.

KHAN, H.H.; BIDDAPPA, C.C.; JOSHI, O.P. A review of Indian work on phosphorus nutrition of coconut. Journal of Plantation Crops, Kasarogod, v.13, n.1, p.11-21, 1985.

OLLIVIER, J. Potassium deficiency symptoms in coconut. Oléagineux, v.48, p.483-484, 1993.

RAIJ, B.van; QUAGGIO, J.A. Métodos de análise química do solo para fins de fertilidade. Campinas: IAC, 1983.31p. (Boletim técnico, 81)

RAIJ, B.van; CANTARELLA, H.; QUAGGIO, J.A.; FURLANI, A.M.C. Recomendções de adubação e calagem para o Estado de São Paulo. 2.ed. Campinas: IAC, 1996. 285p. (Boletim técnico, 100)

ROGNON, F. Cocotier. In: MARTIN-PRÉVEL, P., GAGNARD, J., GAUTIER, P. (Eds.) L'analyse végétale dans le contrôle de l'alimentation des plantes tempérées et tropicales. Paris: Tec\&Doc, 1984. p.447-57.

SAES, L.A. Resposta da bananeira "nanicão" à calagem na região do Vale do Ribeira. Piracicaba, 1995. 82p. Dissertação (Mestrado em Agronomia) - Escola Superior de Agricultura Luiz de Queiroz, Universidade de São Paulo.

SANCHES, A.C.; SILVA, A.P.; TORMENA, C.A.; RIGOLIN, A.T. Impacto do cultivo de citros em propriedades químicas, densidade do solo e atividade microbiana de um podzólico vermelho-amarelo. Revista Brasileira de Ciência do Solo, Viçosa, v.23, p.91-99, 1999.

SOBRAL, L.F. Nutrição e adubação do coqueiro. In: FERREIRA, J.M.S.; WARWICK, D.R.N.; SIQUEIRA, L.A. A cultura do coqueiro no Brasil. 2.ed. Aracaju: EMBRAPA-CPATC,1998. P.129-157.

SOBRAL, L.F.; SANTOS, Z.G. Sistema de recomendações de fertilizantes para o coqueiro (Cocos nucifera $L$.) com base na análise foliar. Brasília: EMBRAPA-DDT, 1987. 23p. (EMBRAPA-CNPCo. Documentos, 7)

TEIXEIRA, L.A.J.; NATALE, W.; RUGGIERO, C. Alterações em alguns atributos químicos do solo decorrentes da irrigação e adubação nitrogenada e potássica em bananeira após dois ciclos de cultivo. Revista Brasileira de Fruticultura, Jaboticabal, v.23, n.3, p.684689, 2001. 